\title{
Commentary Volume, outcome, and the organization of intensive care
} Jeremy M Kahn ${ }^{1,2}$

\author{
1Division of Pulmonary, Allergy \& Critical Care, University of Pennsylvania School of Medicine, 723 Blockley Hall, 423 Guardian Drive, Philadelphia, \\ PA 19104, USA \\ 2Leonard Davis Institute of Health Economics, 3641 Locust Walk, University of Pennsylvania, Philadelphia, Pennsylavania 19104 USA
}

Corresponding author: Jeremy M Kahn, jmkahn@mail.med.upenn.edu

Published: 3 May 2007

Critical Care 2007, 11:129 (doi:10.1186/cc5776)

This article is online at http://ccforum.com/content/11/3/129

(C) 2007 BioMed Central Ltd

See related research by Peelen et al., http://ccforum.com/content/11/2/R40

\begin{abstract}
Increasing evidence suggests that high case volume is associated with improved outcomes in the intensive care unit (ICU). Potential explanations for the volume-outcome relationship include selective referral, clinical experience and organizational factors common to high-volume ICUs. Distinguishing between these explanations has important health policy implications, because outcomes at lowvolume ICUs could be improved either by exporting best practices found at high-volume centers or by regionalizing adult critical care two very different care strategies. Future research efforts should be directed at better characterizing the process of care in high-volume ICUs and exploring the feasibility of creating a regionalized system of care.
\end{abstract}

\section{Introduction}

In the previous issue of Critical Care, Peelen and colleagues add to the growing body of literature demonstrating that increased volume is associated with improved outcomes in the intensive care unit (ICU) [1]. In a large national cohort, patients with sepsis admitted to high-volume ICUs experienced a significant reduction in the adjusted odds of death compared with patients in low-volume ICUs. The association persisted after adjusting for severity of illness and other organizational factors associated with mortality. Of all the potential factors examined, the only other organizational characteristic associated with the outcome of patients with sepsis was the presence of a medium care unit, a finding that may be an artifact of discharge practices.

To date there are now six published studies directly examining the volume-outcome relationship in the ICU, in addition to the many studies examining trauma and high-risk surgery, which frequently involve intensive care [2-7]. Each of these studies examines different patient populations and uses a different threshold for defining a high-volume center, making a formal meta-analysis impossible. Nonetheless, there is an impressively consistent effect: nearly all studies using clinical risk adjustment demonstrate an improvement in outcome with increasing caseload. Given the wealth of evidence, it is now time to take a deeper look into the mechanism behind the volume-outcome effect and attempt to translate this knowledge into improved care for patients.

\section{Understanding the volume-outcome relationship in the ICU}

The classic explanations for the volume-outcome effect are either clinical experience (namely, 'practice makes perfect') or selective referral (the concept that patients are naturally referred to centers of excellence). In the ICU, there is an additional level of complexity. Multiple organizational factors are thought to be associated with improved outcome in critical care, including multidisciplinary rounds, the presence of a clinical pharmacist, care protocols for weaning and sedation, nurse staffing and education, and a culture of teamwork and communication [8]. It is possible that highvolume ICUs are more likely to have these structures in place, independently of clinical experience or selective referral. Translation of new evidence into practice may also have a role, if high-volume centers are better at adopting potentially lifesaving therapies such as low-tidal-volume ventilation for acute lung injury or early adequate resuscitation for sepsis [9].

Distinguishing between all of these potential mechanisms is not simply academic, because there are vastly different health policy implications for each. If the issue is care protocols and evidence-based bundles that can easily be exported to small ICUs, efforts can be directed to expanding the use of these practices. If the issue is caregiver experience, then perhaps the best solution is to regionalize critical care in a manner similar to that for trauma or neonatal care [10]. Regionalization offers the possibility of expanding access to

$\mathrm{ICU}=$ intensive care unit. 
high-quality critical care by bringing critically ill patients from small hospitals to large regional care centers. However, transfer of critically ill patients is not without risks, and little is known about the feasibility of regionalization in terms of costs, distances between hospitals, and number of potential lives saved.

If, in contrast, the benefit of high volume lies in staffing-related attributes such as a multidisciplinary team approach, nursing intensity, or organizational culture, the problem becomes even harder. In theory, small ICUs could adopt new staffing models and hire more nurses. In practice, however, these things may be difficult, if not impossible, to achieve; experienced nurses and pharmacists are expensive and in short supply. Improving organizational climate seems attractive, yet data on how to improve climate, and whether climate can even be improved at all, are completely lacking. Much more information is needed before teamwork and culture can be viewed as appropriate targets in the struggle to improve outcomes in the ICU.

\section{Where do we go from here?}

Given the broad range of potential explanations for the volume-outcome relationship, several next steps are needed. First, future observational research should attempt to explain the volume-outcome effect through a comprehensive examination of the relationship between ICU structure and outcome. In this regard, the Peelen study is an excellent example of how to integrate survey results with multicenter outcome data to answer these types of research question. Second, policy makers should prioritize efforts to improve critical care in small, community ICUs. Defining and exporting best practices through education, outreach, and, if necessary, regulation must be part of the health policy agenda for critical care. Third, it is time for health systems to start seriously considering the formal regionalization of critical care through the creation of a tiered hospital system. Questions about the costs and benefits of regionalization necessitate careful research and quality measurement as we proceed. However, the continued wide variation in risk-adjusted outcome across hospitals makes it untenable not to consider every option to improve care in the ICU.

\section{Competing interests}

The author declares that they have no competing interests.

\section{Acknowledgements}

The author would like to thank Hannah Wunsch for reviewing a draft of this commentary.

\section{References}

1. Peelen L, De Keizer NF, Peek N, Scheffer GJ, Van der Voort PH, De Jonge $E$ : The influence of volume and ICU organization on hospital mortality in patients admitted with severe sepsis: a retrospective multicenter cohort study. Crit Care 2007, 11: R40.

2. Jones J, Rowan K: Is there a relationship between the volume of work carried out in intensive care and its outcome? Int J Technol Assess Health Care 1995, 11:762-769.
3. Durairaj L, Torner JC, Chrischilles EA, Vaughan Sarrazin MS, Yankey J, Rosenthal GE: Hospital volume-outcome relationships among medical admissions to ICUs. Chest 2005, 128: 1682-1689.

4. Glance LG, Li Y, Osler TM, Dick A, Mukamel DB: Impact of patient volume on the mortality rate of adult intensive care unit patients. Crit Care Med 2006, 34:1925-1934.

5. Kahn JM, Goss CH, Heagerty PJ, Kramer AA, O'Brien CR, Rubenfeld GD: Hospital volume and the outcomes of mechanical ventilation. $N$ Engl J Med 2006, 355:41-50.

6. Needham DM, Bronskill SE, Rothwell DM, Sibbald WJ, Pronovost PJ, Laupacis A, Stukel TA: Hospital volume and mortality for mechanical ventilation of medical and surgical patients: a population-based analysis using administrative data. Crit Care Med 2006, 34:2349-2354.

7. Halm EA, Lee $C$, Chassin MR: Is volume related to outcome in health care? A systematic review and methodologic critique of the literature. Ann Intern Med 2002, 137:511-520.

8. Carmel S, Rowan K: Variation in intensive care unit outcomes: a search for the evidence on organziational factors. Curr Opin Crit Care 2001, 7:284-296.

9. Kahn JM, Rubenfeld GD: Translating evidence into practice in the intensive care unit: the need for a systems-based approach. J Crit Care 2005, 20:204-206.

10. Barnato $A E$, Kahn JM, Rubenfeld GD, McCauley K, Fontaine $D$, Frassica JJ, Hubmayr R, Jacobi J, Brower RG, Chalfin D, et al: Prioritizing the organization and management of intensive care services in the United States: the PrOMIS Conference. Crit Care Med 2007, 35:1003-1011. 